\title{
The Super Bug Staph: Vancomycin and Alternative Therapy
}

\author{
Asim Ahmed Elnour ${ }^{1 *}$, Deema AL Hashmi² ${ }^{2}$ Zakieh Mahmoud Alchikh ${ }^{2}$ and Akshaya Srikanth ${ }^{3}$ \\ ${ }^{1}$ Associate Professor. Pharmacy program, Fatima College of Health Sciences-FCHS, UAE \\ ${ }^{2} 4$ th Year, Pharmacy program, FCHS, Abu Dhabi Campus, Al Ain UAE \\ ${ }^{3}$ Department of Clinical Pharmacy, College of Medicine and Health Sciences, Gondar University, Gondar, Ethiopia
}

\begin{abstract}
Received: April 30, 2016; Accepted: May 03, 2016; Published: May 09, 2016
*Corresponding author: Asim Ahmed Elnour*, Associate Professor. Pharmacy program, Fatima College of Health Sciences-FCHS, UAE, Tel: +971525437665; E-mail: asim.ahmed@fchs.ac.ae
\end{abstract}

Since more than 60 years and Vancomycin continues to be in clinical use despite the enormous Concerns of, emergence of resistance and toxicity. These pitfalls are more clinically relevant and resemble real barriers to optimal therapeutic utility of the old glycopeptide. The clinical use of Vancomycin has been associated with resistant Vancomycin resistant staphylococcus aureus-VRSA, Vancomycin resistant enterococciVRE and Vancomycin-resistant Enterococcus faecium (VREF), nephrotoxic effect, patient discomfort, nursing time and the need for therapeutic drug monitory (with debatable trough levels) that limited its clinical utility. In reviewing the literature of one of the most widely used drug for treatment of staph and enterococcus infections, there were suitable alternatives to Vancomycin that should be incorporated into hospital formularies and guidelines with respective evidence-based data. These include Linezolid, Tidizolid, Daltavancin/Oriltavancin, Daptomycin, Tigacycline, Quinupristin/Dalfopristin and Ceftobiprole/Ceftaroline (novel Cephalosporins). The new remedies with specific clinical utility are becoming available and offer better tolerability profile, successful cure rate and minimal rates of resistance. The use of alternative drugs such as [Linezolid, Tidizolid, Daltavancin/ Oriltavancin, Daptomycin, Tigacycline, Quinupristin/Dalfopristin (Streptogramin), Ceftobiprole/Ceftaroline (Cephalosporins)], need to be delineated with specific guidelines where appropriate. Hospitals should have these alternatives available to their formularies with very restrictive guidelines and policies instituted in place to guide the use of these valuable drugs and avoid indiscriminate utilization and emergence of resistance. Linozolid covers acute bacterial skin, skin structure infectionsABSSSI and pneumonia caused by MRSA and VRE.Tidezolid (oral and injectable) which is recently approved in 2014 confers more coverage of ABSSSI caused by MRSA, various Streptococcus species and Enterococcus faecalis. Both drugs do not require renal dose adjustment. Daptomycin approved in 2003 for ABSSSI and endocarditis caused by MRSA can be a suitable alternative to Vancomycin where appropriately indicated. Tigacycline is another possible alternative in vancomycin susceptible enterococcus faecalis. Quinupristin/Dalfopristin introduced in 1999 is active against VREF bacteremia with no renal dose adjustment but its pharmacokinetics is not well studied. A new novel molecule of Dalbavancin approved in 2014 for ABSSSI caused by MRSA and Streptococcus pyogenes infections with one or two doses regimen followed by one dose after one week. A superb similar drug named Oritavancin approved in 2014 covers ABSSSI caused by Staphylococcus aureus, MRSA, enterococci, streptococci and Clostridium difficile with a single dose administered over 3 hours infusion. Both drugs need reduced dosing in renal impairment. We highly recommend the deployment of these alternatives and express our great appreciations for Vancomycin.

\section{References}

1. Liu C, Bayer A, Cosgrove SE, Daum RS, Fridkin SK, Gorwitz RJ, et al. Clinical practice guidelines by the infectious diseases society of america for the treatment of methicillinresistant Staphylococcus aureus infections in adults and children: executive summary.2011;52(3):285-292. doi: 10.1093/cid/cir034.

2. Rybak M, Lomaestro B, Rotschafer JC, Robert Moellering Jr, William Craig, Marianne Billeter, et al. Therapeutic monitoring of vancomycin in adultpatients: a consensus review of the American Society of Health-System Pharmacists, theInfectious Diseases Society of America, and the Society of Infectious Diseases Pharmacists. American Journal of Health-System Pharmacy. 2009;66(1):82-98. doi:10.2146/ ajhp080434.

3. Locke JB, Zurenko GE, Shaw KJ, Bartizal K. Tedizolid for the management of human infections: in vitro characteristics. Clin Infect Dis. 2014;58:S35-S42. doi:10.1093/cid/cit616.

4. Clinical and Laboratory Standards Institute (CLSI). Performance Standards for Antimicrobial Susceptibility Testing; Twenty-fourth Informational Supplement, CLSI document M100-S24, Clinical and Laboratory Standards Institute, 950 West Valley Road, Suite 2500, Wayne, Pennsylvania 19087, USA, 2014. 